\title{
Comparison of Tubeless Mini Percutaneous Nephrolithotomy with Conventional Technique in a Tertiary Care Center
}

\author{
Gupta DK ${ }^{1}$, Gnyawali $A^{2}$, Jaiswal D²
}

\begin{abstract}
Introduction: Mini Percutaneous Nephrolithotomy (mPCNL) is a safe and efficient method for management of nephrolithiasis. Post procedure nephrostomy tube drainage is considered as the standard practice. In recent years, tubeless mPCNL with the use of double J (DJ) stent alone has replaced the placement of the nephrostomy tube. Aims: This study intends to evaluate the safety and efficacy of tubeless Mini Percutaneous Nephrolithotomy. Methods: A total of 80 patients with Nephrolithiasis, admitted to Urology Unit of Nepalgunj Medical College, between September 2018 and September 2019 were enrolled in the study and divided into two groups: Tubeless group where tube was omitted and Standard Group where it was placed. The two groups were compared with respect to hemoglobin drop and blood transfusion requirement, hospital stay and analgesic requirement in the post-operative period. Results: Mean age of the patients was $34.30 \pm 13.19$ years. Mean stone size was $19.03 \mathrm{~mm}$. The mean change in hemoglobin after standard mPCNL was $1.68 \mathrm{gm} / \mathrm{dl}$ and that in the tubeless group was 1.11 ( $p=0.018$ ). The tubeless group had a significantly $(p=0.001)$ shorter hospital stay ( $3.05 \pm 1.23$ days) compared to standard group ( $3.85 \pm 0.86)$. The postoperative pain as assessed by visual analogue scale, was more in the standard group necessitating additional analgesia. It was significantly higher in the standard group at 12, 24, 48 hours, as compared to the tubeless group. Conclusion: Placement of nephrostomy tube can be omitted as a routine practice as Tubeless mini PCNL has an added advantage of significantly reduced postoperative pain, less analgesic requirement, shorter hospital stay, less postoperative blood loss.
\end{abstract}

Keywords: Mini-perc, Mini-percutaneous nephrolithotomy, Tubeless

Authors:

1. Dr. Dipesh Kumar Gupta

2. Dr. Arun Gnyawali

3. Dr. Deepak Jaiswal

${ }^{1}$ Department of Uro-Surgery, Nepalgunj Medical College and Teaching Hospital, Nepalgunj, Banke

${ }^{2}$ Department of Surgery, Nepalgunj Medical College and Teaching Hospital, Nepalgunj, Banke

\section{Address for Correspondence:}

Dr.Dipesh Kumar Gupta

Associate Professor

Department of Uro-Surgery

Nepalgunj Medical College

Nepalgunj, Banke

Email: drdipeshgupta@gmail.com

\section{INTRODUCTION}

Percutaneous nephrolithotomy (PCNL) after its introduction in $1976^{1}$, has rapidly evolved into gold standard for treatment of greater size range of renal stones. Miniaturization has further helped in managing renal stones with smaller sized sheaths without compromising stone free rate. Mini PCNL (mPCNL) provides excellent surgical outcomes and reduced incidence of surgical complications. ${ }^{2}$ Traditionally, a nephrostomy drainage tube and a ureteric double-J stent are placed during PCNL surgery. The purpose of this indwelling nephrostomy tube is to provide adequate urinary drainage, hemostatic tamponade of the access tract, and preserve renal access for a possible second-look PCNL. However, the need for placing a nephrostomy tube has been challenged by several authors since 1997.3 Many reports have confirmed the safety and efficacy of tubeless PCNL, and demonstrated the benefits of a lower analgesic requirement and earlier hospital discharge with no increase in morbidity. ${ }^{3-6}$ Considering similar safety profile of Tubeless $\mathrm{mPCNL}$ as compared to standard MPCNL, and paucity of similar studies conducted in our region, we intended to evaluate the safety of Tubeless mini-PCNL in our Institute.

\section{METHODS}

A total of 80 patients with Nephrolithiasis, admitted to Urology Unit of Nepalgunj Medical College, Banke between September 2018 and September 2019 were enrolled in the study. Ethical clearance for the study was obtained from Institutional review board. Patients with solitary stones less than $3 \mathrm{~cm}$ in size were included and divided into two groups: Tubeless group where 
nephrostomy tube was omitted and Standard Group where it was placed. Intravenous urography (IVU) or CT urography (CTU) was done to evaluate the stone characteristics, renal anatomy and other visceral condition. Stone dimension was recorded in millimeter $(\mathrm{mm})$ along its maximum length. Urine culture and sensitivity test was done and those with negative cultures were taken for the procedure. Mini PCNL was done as a standard procedure using $15 \mathrm{Fr}$ or $18 \mathrm{Fr}$ sheath under fluoroscopic guidance. Pneumatic lithotripter was used for fragmentation of the stones. Nephrostomy tube placement was predetermined based on odd or even inpatient number. Per operative and post-operative complications were recorded. Patient was rendered stone free once it was not visible in plain X-ray KUB done in post-operative day two. For pain management, all patients were given intravenous dose of Ketorolac (30 mg, 8 hourly) as baseline analgesic. Patients requiring additional analgesia were given a single intravenous dose of Tramadol (50 mg, SOS). Postoperative pain was assessed using the Visual Analogue Scale Score. Nephrostomy Tube, in the standard group was removed on $2^{\text {nd }}$ day. The two groups were compared with respect to hemoglobin drop and blood transfusion requirement, hospital stay and analgesic requirement in the post-operative period.

IBM SPSS (version 20.0) was used for analysis of data. Chi square and Independent t-Test was applied where applicable, and $p$ value of $<0.05$ was considered significant.

\section{RESULTS}

There were 49 females and 31 males in the study. The standard group had 27 Females and 13 Males while the tubeless group had 22 Females and 18 Males. The patients enrolled in this study ranged from 17 - 74 years with $43.8 \%$ patients being between 15 to 30 years. The mean age was $34.30 \pm 13.19$ years. The mean age was comparable in both the groups $(p=0.89)$ with $34.15 \pm 13.12$ years and $34.15 \pm 13.43$ years in standard and tubeless groups respectively (Table I).

Patients had primary renal stones ranging from $1-3 \mathrm{~cm}$. The mean stone size was $19.03 \mathrm{~mm}$. The mean stone size in the standard group was $20.61 \mathrm{~mm}$ and in the tubeless group, it was $17.45 \mathrm{~mm}$. Majority of patients had stones involving the right kidney (58\%).

\begin{tabular}{|lccc|}
\hline \multicolumn{1}{|c}{ Variables } & $\begin{array}{c}\text { Tubeless } \\
\text { Group }\end{array}$ & $\begin{array}{c}\text { Standard } \\
\text { Group }\end{array}$ & p value \\
\hline Sex & 18 & 13 & \\
$\quad$ Male & 22 & 27 & 0.25 \\
$\quad$ Female & $1.2: 1$ & $2.07: 1$ & \\
$\quad$ Female : Male & 34.48 & 34.08 & 0.89 \\
\hline Mean Age (Years) & 17.53 & 19.43 & 0.09 \\
\hline Mean Stone Burden (mm) & & & \\
Stone Location & 6 & 3 & \\
$\quad$ Upper Calyx & 5 & 7 & 0.62 \\
$\quad$ Middle Calyx & 15 & 13 & \\
$\quad$ Lower Calyx \\
$\quad$ Renal Pelvis
\end{tabular}

Table l: Comparison of patient characteristics between Tubeless and Standard groups
Approaches to the kidney in both the groups were not significant statistically with a $\mathrm{p}$ value of 0.411 . Thirty two patients in total were approached subcostally. Twelve patients and 36 patients had supra $11^{\text {th }}$ and supra $12^{\text {th }}$ approach respectively. Single tract access was successful in all the cases (Table II).

\begin{tabular}{|cccc|}
\hline Variables & $\begin{array}{c}\text { Tubeless } \\
\text { Group }\end{array}$ & $\begin{array}{c}\text { Standard } \\
\text { Group }\end{array}$ & P Value \\
\hline $\begin{array}{l}\text { Sheath Size (Fr) } \\
\text { Mean }\end{array}$ & 16.35 & 16.90 & 0.12 \\
15 & 22 & 16 & 0.18 \\
18 & 18 & 22 & \\
20 & 0 & 2 & \\
$\begin{array}{l}\text { Approach } \\
\quad \text { Subcostal }\end{array}$ & 16 & 16 & 0.41 \\
$\quad \begin{array}{l}\text { Supracostal } \\
\text { Supra11 }\end{array}$ & 20 & 16 & \\
$\begin{array}{l}\text { Mean Operative } \\
\text { Time(min) }\end{array}$ & 4 & 8 & 0.12 \\
Haemorrhage & 35.13 & 41.88 & \\
\hline
\end{tabular}

Table II: Comparison of intra-operative parameters between Tubeless and Standard groups

Stone clearance was comparable in both groups, however complications were more in standard group. Apart from pain, a total of 18 patients had minor intraoperative complications. Overall, the complications were observed to be more in the standard MPCNL group (Table III).

\begin{tabular}{|lccc}
\multicolumn{1}{c}{ Variables } & Tubeless & Standard & P Value \\
\hline Stone Clearance & $100 \%$ & $100 \%$ & - \\
\hline Complications & 5 & 13 & 0.032 \\
Hematuria & 1 & 7 & \\
Fever & 3 & 2 & \\
Headache & 0 & 1 & \\
SOB & 1 & 0 & \\
Pneumothorax & 0 & 1 & \\
$\quad$ Soakage & 0 & 2 & 0.0018 \\
Hb Change (gm/dl) & $1.11 \pm 1.017$ & $1.68 \pm 1.104$ & 0.040 \\
Blood Transfusion (N) & 0 & 4 & 0.001 \\
\hline Hospital stay (days) & $3.05 \pm 1.23$ & $3.85 \pm 0.86$ & \\
\hline
\end{tabular}

Table III: Comparison of post-operative parameters between Tubeless and Standard groups

Tubeless MPCNL had significantly lesser VAS as compared to standard mPCNL at different periods of time (Table IV). Additional analgesia was required in significant number of patients in the standard MPCNL group. An additional analgesic dose of Intravenous Tramadol $50 \mathrm{mg}$ at 12 hours was needed in $67.5 \%$ of patients in the tubeless group and $87.5 \%$ of patients in standard group $(p=0.032)$.

\begin{tabular}{|cccc}
$\begin{array}{c}\text { Visual Analogue } \\
\text { Score }\end{array}$ & Tubeless mPCNL & $\begin{array}{c}\text { Standard } \\
\mathrm{mPCNL}\end{array}$ & $\mathrm{p}$ Value \\
\hline $\mathbf{1 2}$ Hours & $6.1 .3 \pm 0.79$ & $6.65 \pm 0.80$ & 0.004 \\
\hline $\mathbf{2 4}$ Hours & $3.58 \pm 0.98$ & $4.00 \pm 0.87$ & 0.045 \\
\hline $\mathbf{4 8}$ Hours & $1.20 \pm 0.79$ & $1.88 \pm 1.04$ & 0.002 \\
\hline
\end{tabular}

Table IV: Comparison of Visual Analogue Score between Tubeless and Standard Groups at 12, 24 and 48 Hours 


\section{DISCUSSION}

Placement of the nephrostomy tube along with a nephroureteric stent or stent alone has been a matter of debate ever since Bellman et al $^{3}$ in 1997 challenged the need of a nephrostomy tube. They suggested that a nephrostomy tract is a controlled trauma, which, if properly drained, should heal spontaneously. This improvement in the procedure makes it feasible and safe with advantage of reduced postoperative analgesic requirement and early return to normal life.

Rifaioglu et al $^{7}$ after a retrospectively designed multicenter study, tried to define criteria for placement of nephrostomy tube. Their indications for tubeless PCNL were minimal bleeding during the case, no evidence of collecting system perforation, and low index of suspicion for residual stone fragments. Similarly, Feng et al in their randomized controlled study found that the tubeless cohort experienced the least morbidity. ${ }^{8}$

In our study, the change in hemoglobin was significantly less in the tubeless group with $1.68 \mathrm{gm} / \mathrm{dl}$ in standard and 1.11 $\mathrm{gm} / \mathrm{dl}$ in the tubeless group. In a study done by Cheng et al, mini-PCNL was associated with less hemoglobin decrease and bleeding necessitating transfusion $(p<0.05)$. They compared 132 patients in the tubeless group with 139 patients in the standard group. In their study the mean drop in hemoglobin was $0.53 \pm 0.79$ in the tubeless group and $0.97 \pm 1.42$ in the standard group. ${ }^{9}$

The postoperative hospital stay was significantly less in tubeless group in our study. Other studies quote similar results. In a study done by Knoll et al, hospital stay was significantly shorter in the tubeless group ( $3.8 \pm 2.8$ days) with $p$ value of $0.021 .{ }^{10}$ Similar observation was made by others where mean duration of hospital stay was less in tubeless group. ${ }^{2,11}$ Patients in tubeless group in our study experienced lesser pain as evidenced by lesser VAS score at 12, 24 and 48 hours. Knoll et al also revealed similar findings where, VAS on postoperative day 1 , was lower after mPCNL (VAS: 3.3 vs 4.3 ; $P=0.048$ ), and patients needed slightly less additional pain medication. ${ }^{10}$ Patients in standard group required more top up analgesia in our study. Other studies show similar results stating lesser need of top up analgesia in tubeless group. ${ }^{2,11}$

\section{LIMITATIONS}

Subgroup analysis of only subcostal approach and consistent sheath size might give comparable results as supracostal approaches might be more painful in certain circumstances and even within mini PCNL larger sheath size e.g. $18 \mathrm{Fr}$ might be more painful as compared to $15 \mathrm{Fr}$.

\section{CONCLUSION}

The clinical efficacy and safety of tubeless and standard mini PCNL are similar in all measurements. Placement of nephrostomy tube can be omitted as a routine practice as Tubeless mini PCNL has an added advantage of significantly reduced postoperative pain, less analgesic requirement, shorter hospital stay, and less postoperative blood loss.

\section{REFERENCES}

1. Fernstrom I, Johansson B. Percutaneous pyelolithotomy. A new extraction technique. Scand J Urol Nephrol. 1976; 10(3): 257-9.

2. Lu Y, Ping JG, Zhao XJ, Hu LK, Pu JX. Randomized prospective trial of tubeless versus conventional minimally invasive percutaneous nephrolithotomy. World J Urol. 2013; 31(5): 1303-7.

3. Bellman GC, Davidoff R, Candela J, Gerspach J, Kurtz S, Stout L. Tubeless percutaneous renal surgery. J Urol. 1997; 157(5): 1578-82.

4. Glover M, Moreira CG, Sperandio V, Zimmern P. Recurrent urinary tract infections in healthy and nonpregnant women. Urol Sci. 2014; 25(1): 1-8.

5. Jou YC, Cheng MC, Lin CT, Chen PC, Shen JH. Nephrostomy tubefree percutaneous nephrolithotomy for patients with large stones and staghorn stones. Urology. 2006; 67(1): 30-4.

6. Akman $T$, Binbay $M$, Yuruk $E$, et al. Tubeless procedure is most important factor in reducing length of hospitalization after percutaneous nephrolithotomy: results of univariable and multivariable models. Urology. 2011; 77(2): 299-304.

7. Rifaioglu MM, Onem K, Buldu I, Karatag T, Istanbulluoglu MO. Tubeless percutaneous nephrolithotomy: yes but when? A multicentre retrospective cohort study. Urolithiasis. 2014; 42(3): 255-62.

8. Feng MI, Tamaddon K, Mikhail A, Kaptein JS, Bellman GC. Prospective randomized study of various techniques of percutaneous nephrolithotomy. Urology. 2001; 58(3): 34550.

9. Cheng F, Yu W, Zhang X, Yang S, Xia Y, Ruan Y. Minimally invasive tract in percutaneous nephrolithotomy for renal stones. J Endourol. 2010; 24(10): 1579-82.

10. Knoll T, Wezel F, Michel MS, Honeck P, Wendt-Nordahl G. Do patients benefit from miniaturized tubeless percutaneous nephrolithotomy? A comparative prospective study. J Endourol. 2010; 24(7): 1075-9.

11. Aghamir SM, Hosseini SR, Gooran S. Totally tubeless percutaneous nephrolithotomy. J Endourol. 2004; 18(7): 647-8. 